\title{
Artigo Original / Original Paper \\ Dinâmicas de conhecimento e uso de plantas medicinais em um assentamento rural de Belém do Pará - PA
}

Dynamics of knowledge and use of medicinal plants in a rural settlement of Belém do Pará - PA

\author{
Paula Maria Correa de Oliveira Melo ${ }^{1,3,4}$, Ronize da Silva Santos ${ }^{2}$ \& Marlia Coelho-Ferreira ${ }^{2}$
}

\begin{abstract}
Resumo
Os agricultores familiares que vivem em áreas de assentamentos rurais estão entre os diversos grupos sociais da Amazônia que detêm um amplo conhecimento sobre o uso de plantas medicinais. Estudos realizados neste contexto revelam uma vasta heterogeneidade no conhecimento e uso de plantas medicinais, bem como a influência dessas comunidades na diversidade vegetal e nos aspectos culturais associados ao seu uso. Esta pesquisa traz informações sobre a dinâmica do conhecimento e uso de plantas medicinais no Assentamento Rural Paulo Fonteles, estabelecido desde o ano de 2006 no Distrito de Mosqueiro, Município de Belém, estado do Pará. Os dados foram obtidos através de entrevistas semiestruturadas e oficinas participativas e analisados qualitativa e quantitativamente. As plantas medicinais são recursos prioritários para os assentados em períodos que antecedem e sucedem a ocupação. Foram registradas 140 plantas medicinais, das quais, 119 foram identificadas e pertencem a 58 famílias botânicas. Sessenta espécies são nativas do Brasil, sendo 21 com domínio fitogeográfico restrito a Amazônia e 59 são introduzidas. O conhecimento local de plantas medicinais encontra-se bem difundido entre informantes de diferentes classes de idade e gênero. As pessoas provenientes de outras regiões do Brasil reportaram um acervo de plantas medicinais significativamente maior do que àquelas naturais do estado do Pará. Entre as 140 plantas mencionadas, 110 referem-se a espécies cujos usos foram mantidos ao longo do tempo pelos informantes. A heterogeneidade sociocultural e a dinâmica do modo de vida dos assentados rurais contribuem para o acúmulo de saberes e no incremento da diversidade da flora local. Palavras-chave: Amazônia brasileira, comunidades assentadas, conhecimento local, etnobotânica.
\end{abstract}

\begin{abstract}
Rural communities that were founded based on land occupation in the Amazon usually have a wide knowledge about the use of medicinal plants. Studies carried out in this context reveal a vast heterogeneity in terms of knowledge and use of medicinal plants. They also describe the influence these communities have on plant diversity and the cultural aspects associated with their use. This paper shows the dynamics of knowledge and use of medicinal plants in the Paulo Fonteles Rural Settlement, established in 2006 in the Mosqueiro District, Belém Municipality, Pará state, Brazilian Amazon. Data was collected through semi-structured interviews and participatory workshops and analyzed qualitatively and quantitatively. Medicinal plants are crucial resources for farmers before and after land occupation. A total of 140 ethnospecies were recorded, of which 119 were identified; They belong to 58 botanical families. 60 species are native to Brazil. Out of those, 21 belong to the phytogeographic domain restricted to Amazonia. 59 species were introduced the region. The local knowledge of medicinal plants is well spread among informants of different age and sex groups. People from other regions in Brazil reported significantly more medicinal plants than those native to the state of Pará. Among the 140 plants mentioned, 110 refer to species whose uses have been maintained over time by informants. The socio-cultural heterogeneity and the dynamic livelihood of the farmers has been crucial for the improvement of their knowledge and the increase of the diversity of the local flora.
\end{abstract}

Key words: Brazilian Amazon, settled communities, local knowledge, ethnobotany.

Veja material suplementar em <https://doi.org/10.6084/m9.figshare.13645655.v1>

\footnotetext{
${ }^{1}$ Museu Paraense Emílio Goeldi, Coordenação de Botânica, Prog. Capacitação Institucional, Av. Perimetral 1901, Terra Firme, 66077-830, Belém, PA, Brasil.

${ }^{2}$ Museu Paraense Emilio Goeldi, Coordenação de Botânica, Av. Perimetral 1901, Terra Firme, 66077-530, Belém, PA, Brasil.

${ }^{3}$ ORCID: <https://orcid.org/0000-0003-0354-8584>

${ }^{4}$ Autor para correspondência: paulacorrea92@hotmail.com
} 


\section{Introdução}

O uso de plantas medicinais integra a identidade cultural dos diferentes povos que habitam a Amazônia, reproduzindo-se através de diferentes gerações. O bioma Amazônico possui uma vasta diversidade de recursos naturais com potencial terapêutico, associado a uma expressiva diversidade sociocultural, resultado da herança indígena, europeia e africana, que caracterizaram os povos e comunidades com visões e saberes peculiares (Berg 2010; Almeida 2011). As comunidades rurais fundadas a partir de ocupações territoriais (assentamentos rurais) representam um destes grupos sociais (Almeida \& Gama 2014). Geralmente formadas por pessoas que vivem em migrações periódicas pelo território brasileiro ou no exterior, podendo se instalar em lugares distintos do seu local de origem e se adaptar a novos ambientes sociais, econômicos e naturais. Neste contexto são transformadas suas crenças, valores, saberes, tecnologia, sistemas de intercâmbio e muitos outros aspectos de suas vidas, incluindo seu reconhecimento e uso dos recursos naturais (Nesheim et al. 2006).

A Amazônia compreende aproximadamente 90\% do total de áreas destinadas a assentamentos rurais no Brasil (Ezzine-de-Blas et al. 2011). Apesar disso, poucos estudos têm se ocupado em investigar o conhecimento sobre o uso de plantas medicinais detido por esses grupos, bem como a influência dessas populações nos diversos aspectos que envolvem a diversidade de plantas no local onde estão inseridos. Estudos etnobotânicos realizados em comunidades assentadas no Bioma do Cerrado brasileiro mostram que o conhecimento e uso de plantas medicinais contribuem para a valorização e conservação da flora nativa (Cunha \& Bortolotto 2011; Tunholi et al. 2013). Esses autores também observaram, que o uso das espécies de maior importância local pode estar relacionado com as experiências dessas pessoas em outros ambientes, pois estas geralmente trazem consigo conhecimento de outros tipos de vegetação. Gavioli (2012), por sua vez, registrou que os moradores de um assentamento rural no estado de São Paulo, ao cultivarem um amplo acervo de espécies vegetais, sejam elas nativas ou exóticas, exercem um importante papel no aumento da diversidade vegetal e agricultura regional.

Outros estudos investigaram como o conhecimento de plantas medicinais se comporta frente à heterogeneidade sociocultural observada nestes grupos e constataram que aspectos sociais (gênero, idade, grau de escolaridade, tempo de residência) e culturais (formas de transmissão do conhecimento e histórico migratório) influenciam diretamente neste saber (Araújo 2009; Oliveira 2010; Cunha \& Bortolotto 2011; Matos 2011; Soldati et al. 2015). A pesquisa de Soldati et al. (2015) junto a assentamentos rurais no sudeste do Brasil mostrou que apesar da instabilidade socioambiental vivida por estas pessoas, o conhecimento sobre plantas medicinais ainda é transmitido de maneira vertical, ou seja, de uma pessoa para muitas. Este padrão também foi registrado no estudo de Araújo (2009) no estado da Paraíba, que, além disso, apontou o uso destes recursos sendo mais comum entre as mulheres, pessoas com maior idade e maior tempo de residência na área estudada. Oliveira (2010), por sua vez, destacou a relevância do histórico migratório dos moradores de um assentamento rural no estado de São Paulo sobre o conhecimento acumulado por cada indivíduo.

Este último aspecto vem sendo abordado em trabalhos, que embora não tenham sido realizados no contexto de assentamentos rurais, estudam a influência dos processos migratórios na dinâmica do uso de plantas medicinais. Estes estudos foram conduzidos junto a populações que migraram de um país para outro (Waldstein 2006; Inta et al. 2008; Volpato et al. 2009) e aquelas migrantes entre as regiões de um país Garcia et al. (2010). Medeiros et al. (2012) constataram, que populações migrantes, em geral, se adaptam à composição vegetal do local onde se estabelecem, seja pela necessidade de envolvimento no novo contexto sociocultural, seja pelas novas doenças acometidas, podendo ser ainda em função de uma possível similaridade florística entre o lugar de origem e o atual; assim como, o ambiente recém ocupado será enriquecido com plantas trazidas por estas populações, enquanto estratégia de manutenção da sua herança cultural.

Este trabalho teve como objetivo contribuir com informações sobre a dinâmica do conhecimento e uso de plantas medicinais por moradores do Assentamento Rural Paulo Fonteles (APF), localizado no município de Belém, estado do Pará, Amazônia brasileira. Para alcançar tais objetivos buscou-se responder às seguintes questões: Quais as plantas medicinais indicadas e como são utilizadas pelos assentados ao longo do tempo?; Que fatores influenciam no conhecimento associado a estes recursos?; Que estratégias são empregadas em relação à utilização de plantas medicinais resultante do processo de migração? 


\section{Material e Métodos}

\section{Área de estudo}

O Assentamento Paulo Fonteles (APF) está localizado à margem direita da estrada Baía do Sol, entre Passagem das Flores e Vila Sucurijuquara, no Distrito de Mosqueiro, cerca de 70 quilômetros do município de Belém do Pará (Fig. 1). Está situado em um ambiente de clima Tropical Úmido com temperatura média de $25,9^{\circ} \mathrm{C}$, umidade relativa do ar de $84 \%$ e média de precipitação anual de 2.800 $\mathrm{mm}$, altitude média de 15 metros acima do nível do mar e cobertura vegetal predominante de floresta ombrófila densa (Pantoja 2010).

O APF ocupa área de 930 ha dividida em 60 lotes, com tamanhos diferenciados que vão de 40 a 80 hectares, nos quais foram construídas as moradias e destinada terra para cada produtor desenvolver suas atividades agrícolas. Em relação aos serviços de saneamento básico, o assentamento como um todo possui apenas estruturas básicas rudimentares.

Durante os primeiros anos de sua implantação, a comunidade era assistida por agentes de saúde, porém atualmente, não contam mais com essa assistência, recorrendo em casos de necessidade, a moradora local Germina da Silva, de 54 anos. Ela aprimorou seus conhecimentos sobre plantas medicinais e suas respectivas técnicas de manipulação em um curso técnico em Saúde do Campo que realizou. Outra opção no local é buscar atendimento nas duas unidades básicas de saúde (UBSs), situadas a cerca de três quilômetros do APF.

\section{Assentamento Paulo Fonteles: processo de ocupação \\ As ações para a implantação do APF se} iniciaram no ano de 2003, quando o Movimento dos Trabalhadores Rurais Sem Terra (MST) organizou um grupo de famílias remanescentes de outros assentamentos já estabelecidos no distrito de Mosqueiro, para realizar a apropriação de uma fazenda improdutiva denominada, Fazenda Baía do Sol. Na ocasião, essa tentativa de ocupação não foi bem-sucedida, mas os ocupantes permaneceram na área, acampados em frente à referida fazenda. Neste período iniciaram-se as negociações entre o MST e os proprietários do latifúndio, o que aumentou as expectativas para o estabelecimento do APF. Isto mobilizou várias famílias, que estavam em outros acampamentos a deslocarem-se para a área de Mosqueiro, ampliando o número de pessoas e fortalecendo o grupo (Pantoja 2010).

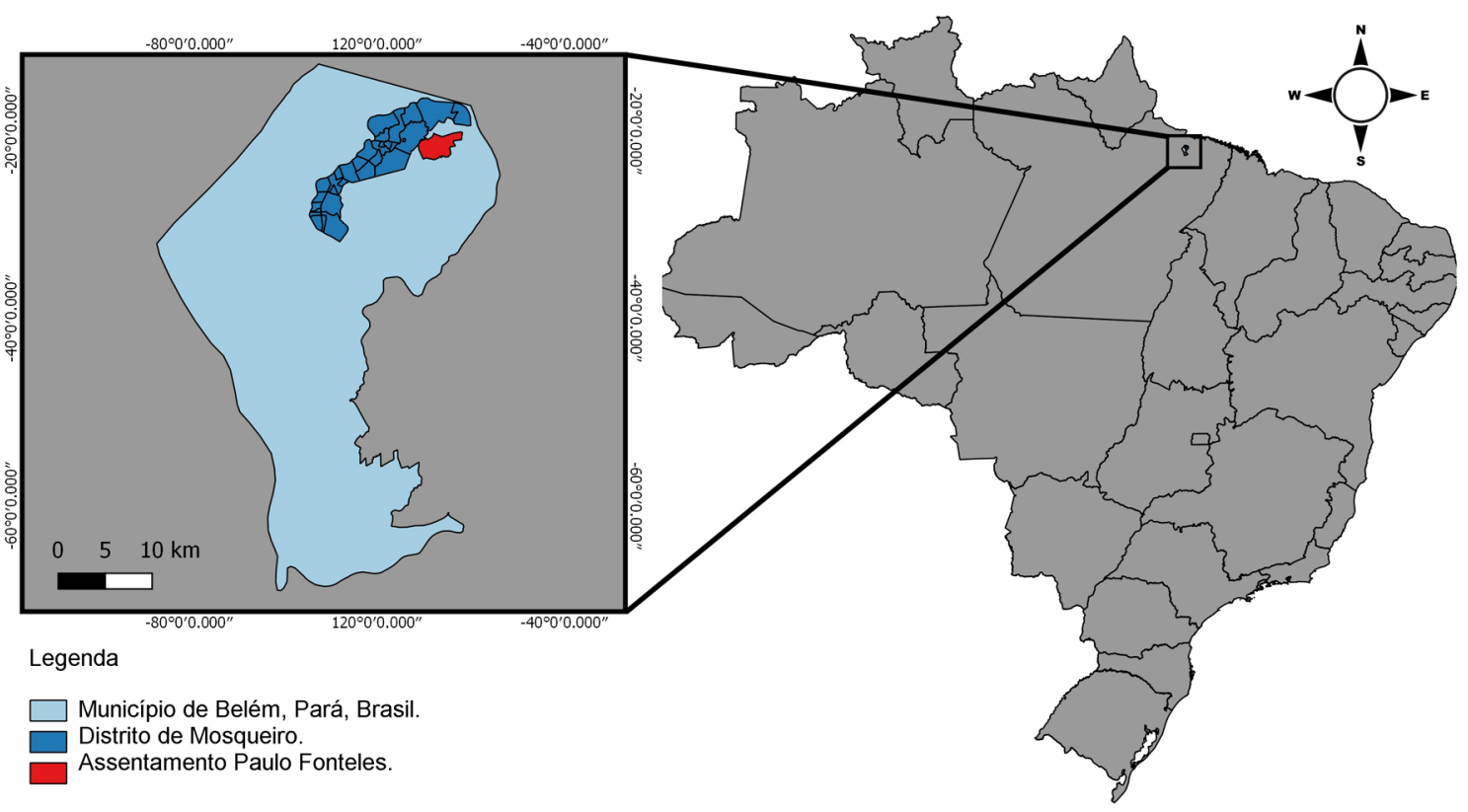

Figura 1 - Mapa de localização do Assentamento Paulo Fonteles, Distrito de Mosqueiro, Município de Belém, estado do Pará, Brasil.

Figure 1 - Map of the Paulo Fonteles Rural Settlement, Mosqueiro District, Belém Municipality, Pará state is located. 
Os acampamentos são organizados pelo MST através de instâncias coletivas de decisões, denominadas de assembleias, e instâncias participativas de gestão e trabalho, chamadas de "coletivos", estes se ocupam de diversos assuntos e ações necessárias ao funcionamento e manutenção da rotina diária do grupo, como saúde, educação, mobilização e segurança (Figueiredo \& Pinto 2014). Durante a fase de acampamento do APF (2003 a 2006), as famílias foram divididas em oito grupos de trabalho ou coletivos e cinco grupos de gestão do assentamento: frente de massas, ciranda infantil, segurança e disciplina, educação e juventude, devendo cada um destes ter um coordenador responsável (Pantoja 2010). Apesar das inúmeras dificuldades vivenciadas neste período, os líderes do MST entendem que esta é uma etapa fundamental para o estabelecimento de um assentamento rural, pois estimula a formação de indivíduos menos passivos diante dos problemas sociais e com maiores possibilidades de desenvolver uma convivência essencialmente coletiva e comunitária (Caldart 2000).

Em 2006, o APF foi estabelecido formalmente, com isso os arranjos iniciais do estilo de vida compartilhado foram rompidos e cada unidade familiar passou a cuidar do seu próprio lote. Esta mudança na estrutura social do grupo pode ser explicada pela realidade complexa da organização interna de um assentamento rural, pois os assentados possuem interesses, por vezes, conflitantes, uma vez que estes possuem maneiras diferentes de planejar e vivenciar as atividades cotidianas dos lotes (Belo \& Pedlowski 2014).

\section{Procedimentos éticos}

O projeto de pesquisa foi apresentado inicialmente a alguns representantes do Assentamento Paulo Fonteles. Na sequência foi realizada uma reunião com os demais moradores, onde foi explicado o projeto para aqueles que compareceram. Nessa ocasião obteve-se o consentimento à realização da pesquisa. As atividades de campo foram realizadas somente depois que os informantes assinaram o Formulário de Consentimento Informado. A pesquisa encontra-se cadastrada no Sistema Nacional de Gestão do Patrimônio Genético e do Conhecimento Tradicional Associado (SisGen), sob o número do cadastro AE259B4, como preconiza a Nova Lei da Biodiversidade, 13,133 de 2015 (Brasil 2015).

\section{Seleção dos informantes}

A comunidade é formada por 55 residências, nas quais habitam aproximadamente 100 indivíduos adultos. Com o intuito de que a pesquisa gerasse dados conclusivos sobre toda a comunidade assentada, buscou-se incluir na amostragem pelo menos um representante de cada unidade familiar residente, com pelo menos 18 anos de idade, interessado em participar. Assim, das 55 unidades familiares situadas no Assentamento, em 46 entrevistou-se o homem ou a mulher e quando possível o casal, que se encontravam disponíveis. Nestas circunstâncias, o universo amostral foi composto por 61 informantes.

\section{Coleta de dados}

Os dados foram obtidos no período entre janeiro e novembro de 2015, totalizando aproximadamente 120 dias de trabalho de campo. As informações foram obtidas através de entrevistas semiestruturadas (Albuquerque et al. 2014) que abordaram os seguintes tópicos: dados pessoais, histórico de migração (nome, sexo, idade, religião, estado civil, local de nascimento, escolaridade, migração e ocupação principal) e dados etnobotânicos (nome da planta, uso, parte usada, modo de preparo, lugar onde a conheceu, ainda a utiliza? existe outra planta que pode ser usada para o mesmo fim? qual?).

A fim de obter informações sobre a influência dos processos que envolveram a formação deste assentamento no uso de plantas medicinais da comunidade, foram realizadas oficinas participativas, ocasião em que os informantes foram estimulados a desenvolver uma linha do tempo de suas vidas (Geilfus 2002). Esse resgate histórico foi dividido em diferentes períodos vivenciados pelos informantes: moradia anterior (10 ou 12 anos atrás), acampamento (2003-2005), assentamento (2006-2010 - período em que os moradores iniciaram a moradia como assentados rurais), ano da pesquisa (2015).

Em cada período foram levantados questionamentos e discussões acerca dos seguintes assuntos: recursos e tratamentos mais frequentemente utilizados nos cuidados com a saúde; doenças mais comuns; as plantas mais usadas e quais as plantas mais abundantes.

Foram realizadas "turnês-guiadas" juntos com os participantes, que consiste em caminhar nos quintais e ambientes florestais do entorno da comunidade, observando as plantas que tem uso 
medicinal pelo entrevistado (Alexiades 1996). A identificação das espécies foi realizada por comparação com a coleção do Herbário MG do Museu Paraense Emílio Goeldi, com o auxílio de técnicos e especialistas desta instituição. Para a identificação das plantas, que foram citadas, mas que não foram encontradas no local de estudo, foram utilizadas fotografias como recurso para estímulos visuais, conforme proposto por Medeiros et al. (2010). Para tanto, primeiramente foram obtidas junto aos entrevistados informações morfológicas, organolépticas (cor, cheiro, sabor, textura), hábito e ambientes de ocorrência das etnoespécies (nomes populares dados a uma ou várias espécies). Em seguida foram apresentadas aos informantes fotografias das prováveis espécies disponíveis na literatura, para o reconhecimento. Por meio deste método, foi possível a identificação ao menos em nível de família ou gênero das etnoespécies.

O sistema de classificação botânica adotado foi o Angiosperm Phylogeny Group - APG IV 2016 (Byng et al. 2016). A nomenclatura botânica foi verificada e atualizada com o auxílio de BFG (2018) e quando necessário, do site do Missouri Botanical Garden (2018) e em seguida checada na base de dados The Plant List (2019). As amostras botânicas férteis foram depositadas no Herbário MG do Museu Paraense Emílio Goeldi.

\section{Análise de dados}

A classificação quanto ao hábito e à origem fitogeográfica das espécies seguiu a base de dados BFG (2018), em que foram consideradas como nativas as espécies, cujos domínios fitogeográficos abrangem a Amazônia e introduzidas/exóticas aquelas de ocorrência natural apenas em outras regiões do país ou do mundo.

Os testes estatísticos foram realizados com base no número de etnoespécies citadas por cada informante. Todos os nomes atribuídos a uma única espécie botânica foram considerados a fim de acomodar toda nomenclatura vernácula empregada na área de estudo, bem como aquelas plantas cuja identificação ocorreu sem material testemunho. As variáveis consideradas nas análises foram sexo, idade e origem dos informantes. A análise de variância (ANOVA) foi utilizada para avaliar a relação entre o número de etnoespécies mencionadas por cada informante e a idade destes, enquanto que o Test $\mathrm{T}$ foi utilizado para verificar se havia diferença significativa no conhecimento entre os grupos de gênero e origem. Para todos esses testes, foi utilizado o software R (Chambers 1998).

A análise de variância para a idade foi realizada, com homens e mulheres distribuídos em cinco classes de idade. Com relação à variável origem foram considerados dois grupos: um de pessoas do estado do Pará e outro grupo das pessoas oriundas de outros estados do Brasil, sendo que ambos contaram com homens e mulheres de diferentes faixas etárias.

Para a organização e análise dos usos atribuídos às etnoespécies foram considerados os termos coletados em campo. A medida em que as etnoespécies foram indicadas, seus usos foram categorizados em: mantidos (planta utilizada para os mesmos fins na localidade anterior e no Assentamento), substituídos (plantas substituídas por outras no atual local de moradia), incorporados (plantas usadas pela primeira vez no APF) ou descontinuados (plantas que são conhecidas, porém não são mais utilizadas). Esta categorização foi empregada no estudo de Garcia et al. (2010).

\section{Resultados e Discussão}

Aspectos sociais dos informantes

No presente estudo, as informantes do sexo feminino constituíram 64\%. A idade dos entrevistados variou entre 18 e 80 anos, com destaque para as classes etárias 28-37 $(24,5 \%)$ e $38-47(27,8 \%)$. Apenas $9,8 \%$ eram analfabetos. Informantes adeptos ao protestantismo ou catolicismo predominaram $(39,3 \%$ e $34 \%$ respectivamente), mas vale ressaltar a representatividade daqueles adeptos ao budismo. Cerca de $65 \%$ dos informantes habitam a área desde que o Assentamento foi fundado, isto é, há pelo menos dez anos. A maior parte dos entrevistados é proveniente da zona rural de outros municípios do estado do Pará $(78,6 \%)$ e os demais vieram de outros estados do Brasil (Maranhão - 9,8\%; Minas Gerais - 3\%; Mato Grosso do Sul - 3\%; Piauí - 1,6\%; Rio Grande do Norte - 1,6\%; e São Paulo - 1,6\%). Com relação ao itinerário migratório, $45,9 \%$ dos informantes possuem histórico definido no plano campo-cidade-campo (Tab. 1).

A agricultura é a principal atividade econômica local, sendo a mandioca (Manihot esculenta Krantz.) a cultura principal destinada principalmente para a produção da farinha. As mulheres, em geral, dedicam-se ao cuidado com a família, a casa, ao cultivo de plantas medicinais, ornamentais e frutíferas nos quintais, além da 
Tabela 1 - Caracterização sociodemográfica de 61 moradores do Assentamento Paulo Fonteles, Distrito de Mosqueiro, Belém, PA.

Table 1 - Sociodemographic data of 61 people in Paulo Fonteles settlement, Mosqueiro District, Belém, PA.

\begin{tabular}{|c|c|c|}
\hline Variáveis & Frequência & $\%$ \\
\hline \multicolumn{3}{|l|}{ Gênero } \\
\hline Masculino & 22 & 36 \\
\hline Feminino & 39 & 64 \\
\hline \multicolumn{3}{|l|}{ Classes de idades } \\
\hline $18-27$ & 6 & 9,8 \\
\hline $28-37$ & 15 & 24,5 \\
\hline $38-47$ & 17 & 27,8 \\
\hline $48-57$ & 13 & 21,3 \\
\hline $58-80$ & 10 & 16,3 \\
\hline \multicolumn{3}{|l|}{ Grau de escolaridade } \\
\hline Iletrado & 6 & 9,8 \\
\hline Ensino fundamental & 33 & 54 \\
\hline Ensino médio & 17 & 27,8 \\
\hline Ensino técnico & 2 & 3,2 \\
\hline Ensino superior & 3 & 5 \\
\hline \multicolumn{3}{|l|}{ Tempo de residência } \\
\hline$<10$ anos & 21 & 34,5 \\
\hline$\geq 10$ anos & 40 & 65,5 \\
\hline \multicolumn{3}{|l|}{ Origem } \\
\hline Estado do Pará & 48 & 78,6 \\
\hline Outros Estados do Brasil & 13 & 21,4 \\
\hline \multicolumn{3}{|l|}{ Religião } \\
\hline Protestantismo & 24 & 39,3 \\
\hline Catolicismo & 21 & 34,4 \\
\hline Budismo & 5 & 8,2 \\
\hline Afro brasileira & 1 & 1,6 \\
\hline Não possuem crença & 10 & 16,3 \\
\hline
\end{tabular}

criação de pequenos animais, como galinhas, patos e porcos. Algumas além de obter renda destas atividades recebem aposentadoria. Os homens por sua vez, declaram-se agricultores e para a maioria esta atividade é a única fonte de renda.

No que se refere ao conhecimento sobre plantas medicinais, $75 \%$ dos informantes afirmaram ter adquirido esses conhecimentos durante a infância junto à família, que inclui pais, mães, avós e sogras. Os demais (25\%) afirmaram ter adquirido este conhecimento, já adultos, com os vizinhos do APF, amigos de outros assentamentos e lugares onde já moraram. Além destas fontes, alguns (8\%) citaram cursos oferecidos pelo MST, livros e revistas, como outras fontes de obtenção desse conhecimento. 
$\mathrm{O}$ uso de plantas medicinais pelos informantes ao longo do tempo Antes da ocupação, 48 (78,6 \%) dos informantes residia em ambiente urbano, e embora preferissem utilizar plantas nos cuidados básicos de saúde, relatam que o acesso a estas era limitado, principalmente, devido à falta de ambiente adequado para o cultivo e o desenvolvimento de atividades fora do ambiente agrícola. Assim, quando necessário recorriam aos medicamentos sintéticos sem prescrição médica.

Durante a fase de acampamento preferiam uso de remédios caseiros, uma vez que estes eram disponibilizados pela comissão responsável pelos cuidados de saúde, integrada por conhecedores de plantas com propriedades terapêuticas. Embora tivessem o direito de ser atendidos nas unidades básicas de saúde do entorno do acampamento, os acampados só recorriam às mesmas, quando a primeira opção se mostrava ineficaz.

Os problemas de saúde mais comuns nesta fase (2003 a 2005) eram gripe, pneumonia, verminoses, vômitos e infecções dermatológicas causadas por fungos e parasitas, os quais associaram à precariedade das estruturas habitacionais temporárias e à alimentação. De acordo com os informantes, nesse período as espécies marupazinho (Eleutherine bulbosa (Mill.) Urb), mangueira (Mangifera indica L), limão (Citrus limon (L.) Osbeck, boldo (Plectranthus sp.) e eucalipto (Eucalyptus sp.) eram as plantas medicinais mais comumente utilizadas. Da mesma forma era utilizado um xarope preparado com pedaços de cupinzeiro, cuja eficácia se atribuía à absorção dos nutrientes das árvores em que se instalava. Desconheciam as plantas de ocorrência natural na área, o que reforçava o uso daquelas facilmente cultivadas nas hortas, como as mencionadas acima.

Depois de se instalarem na área com o novo estatuto de Assentamento Rural, os coletivos deixaram de existir e a maneira de cuidar da saúde foi alterada. Neste período, a Pastoral da Criança - organismo de ação social da Conferência Nacional dos Bispos do Brasil (CNBB) - fornecia atendimento especial a este grupo, verificando que muitas crianças, naquele período se encontravam abaixo do peso.

Foram escolhidos moradores a serem capacitados como Agentes Comunitários de Saúde (ACSs). Durante quatro anos, os ACSs fizeram visitas semanais aos assentados, fornecendo instruções de higiene e limpeza, distribuindo hipoclorito para o tratamento da água e medicamentos industrializados quando necessário. Nos casos mais graves, os pacientes eram encaminhados à Unidade Básica de Saúde (UBS), mais próxima.

Na UBS, os assentados contavam com o atendimento de um médico, que prescrevia, prioritariamente, tratamentos naturais, o que the valeu o apelido de "Dr. Copaíba". Neste período, parte dos informantes recorria primeiramente ao posto de saúde ou medicamentos sintéticos, pela mesma razão mencionada para a fase anterior, mas também por ainda não terem iniciado o cultivo daquelas que conheciam. Outros ainda preferiram o tratamento natural, e quando não sabiam qual planta utilizar para determinado problema, recorriam à Dona Germina.

Atualmente, $79 \%$ dos informantes utilizam plantas como primeira alternativa terapêutica. Esses recursos são obtidos por meio do cultivo nos quintais e do extrativismo na área de florestal do entrono da comunidade. $\mathrm{O}$ atendimento convencional de saúde, que continua sendo realizado na UBS local, é procurado em última instância, por preferirem o tratamento natural e pelos serviços prestados pela unidade ser precário e insuficiente, dada a grande demanda, segundo relato da maioria dos participantes. Revelam que, além disso, os médicos prescrevem dietas e medicamentos que não são adequadas a sua realidade cultural e econômica.

Os males mais frequentes são gripe, cansaço, asma, diarreia; o que não diferem muito de quando ainda estavam na fase de acampamento. As plantas medicinais mais utilizadas são açaizeiro (Euterpe oleracea Mart), algodão-roxo (Gossypium arboreum L.) capim-santo (Cymbopogon citratus (DC.) Stapf), noni (Mansoa alliacea (Lam.) A.H.Gentry), mangueira, hortelãzinho (Anacardium occidentale L.), limão, boldo, goiabeira (Psidium guajava $\mathrm{L}$ ), citronela (Cymbopogon nardus (L.) Rendle), mastruz (Dysphania ambrosioides (L.) Mosyakin \& Clemants), pirarucu (Kalanchoe pinnata (Lam.) Pers.) e urucum (Bixa orellana L.), consideradas acessíveis e versáteis.

A partir da linha do tempo realizada com os participantes foi possível observar que os diferentes períodos vivenciados pelos informantes antes e durante o processo de ocupação influenciaram diretamente em suas opções terapêuticas. Observouse, ainda, que as plantas medicinais constituíram um importante recurso em todos os períodos supracitados. Isto teria sido possível, devido à valorização do uso de plantas que a atividade 
agrícola proporciona, e aos aspectos simbólicos e culturais (Ferreira Júnior et al. 2013), que possivelmente, muitos dos assentados carregam consigo.

Estudo realizado em um assentamento rural no Município de Imbaú (Paraná) relacionou o contínuo uso de plantas medicinais e até mesmo a preferência a estas aos aspectos econômicos destas populações (Visbiski et al. 2003). Em outra pesquisa, observouse que além destes aspectos, a manutenção desta prática nestas áreas se deve principalmente a questões culturais (Scopinho 2010).

\section{Riqueza e composição florística} de plantas medicinais

Um total de 76 indicações terapêuticas foi mencionado para 140 plantas, das quais 138 foram identificadas, 119 em nível de espécie e 19 até gênero. As espécies estão distribuídas em 120 gêneros e 58 famílias, das quais, Asteraceae, Fabaceae e Lamiaceae foram as mais representativas (Tab. S1, disponibilizada no material suplementar $<$ https://doi.org/10.6084/m9.figshare.13645655. v1>). A riqueza de espécies registrada pode ser considerada alta quando comparada as que foram inventariadas em estudos com outros grupos de assentados rurais no Brasil (Visbiski et al. 2003; Araújo 2009; Guerra et al. 2010; Oliveira 2010; Almeida \& Gama 2014; Brito et al. 2017b), nos quais foram identificadas entre 30 e 105 espécies de plantas medicinais.

Entre as 119 plantas identificadas em nível de espécie, 60 são nativas do Brasil com ocorrência na Amazônia, sendo 21 destas com distribuição restrita para este Bioma e três endêmicas do Brasil, são elas cupuaçu (Theobroma grandifolium (Willd. ex Spreng.) K.Schum), barbatimão (Connarus perrottetii var. angustifolius Radlk) e uxi (Endopleura uchi (Huber) Cuatrec.). 59 espécies são de ampla distribuição ou exóticas. A incidência do uso de espécies amazônicas era esperada, considerando que grande parte dos moradores é proveniente de áreas rurais nesse Bioma. Além disso, os entrevistados oriundos de outros estados e biomas demonstraram amplo conhecimento sobre estas plantas, o que indica um considerável compartilhamento de informações sobre a flora local entre essas pessoas. A indicação de um número expressivo de espécies nativas, especialmente as arbóreas, também aproveitadas para fins madeireiros na Amazônia, revela a importância do uso medicinal associado à estas espécies para a conservação delas. Um exemplo disso é Vouacapoua americana (acapú), espécie ameaçada de extinção (Livro vermelho da flora do Brasil 2013) cujas cascas são empregadas localmente no tratamento de inflamações e mioma, tal aspecto contribui localmente como uma barreira para a exploração de sua madeira.

Resultados semelhantes foram observados por Cunha \& Bortolotto (2011) quando realizavam estudo em um assentamento rural no Mato Grosso do Sul, onde $54 \%$ das plantas medicinais levantadas eram nativas do Cerrado, os autores destacaram que este uso contribui para a conservação da flora nativa. De acordo com Tunholi et al. (2013), a utilização de plantas nativas por assentados tende a aumentar à medida que os residentes aumentam o seu conhecimento sobre a vegetação local. E, de acordo com os resultados obtidos em sua pesquisa isto contribui para a conservação, dada a importância de seus derivados para a alimentação e medicina local. Por outro lado, o número quase equivalente de espécies introduzidas, também demonstra um aumento na diversidade vegetal local, fenômeno observado por Gavioli (2012).

Dentre as espécies mencionadas, verônica (Dalbergia monetaria L.) foi a que recebeu o maior número de citações $(\mathrm{n}=28)$. Trata-se de uma liana nativa do bioma amazônico, facilmente encontrada na área de várzea local, cuja casca e partes do lenho são utilizados no preparo de garrafadas, destinadas a tratar dor de barriga, dor na coluna, infecção urinária, úlcera, gastrite, diarreia, ferimentos, anemia e problemas nos órgãos genitais femininos. Estas duas últimas enfermidades receberam destaque e são comumente tratadas com esta espécie em outras comunidades amazônicas (Martins et al. 2005; Freitas \& Fernandes 2006; Coelho-Ferreira 2009). A versatilidade de uso desta espécie, assim como a sua abundância na vegetação local, segundo a concepção dos informantes, talvez explique o seu destaque nesta pesquisa.

\section{Fatores que influenciam}

no conhecimento das plantas medicinais

Os testes estatísticos utilizados para verificar se a idade, o gênero ou a origem influenciam no conhecimento de plantas medicinais entre os informantes, apontaram diferenças significativas apenas entre os grupos categorizados de acordo com a origem (Anova ${ }^{\text {idade: }} \mathrm{f}=0,468, \mathrm{p}>0,05$; Teste tgênero: $t=-0,13493, p>0,05$; Teste torigem: $\mathrm{t}=2.5885, \mathrm{p}<0,05)$. Esta variável apontou que as pessoas oriundas de outros estados apresentam uma superioridade na média do número de plantas 
reportadas em relação às nativas do Pará. Os resultados desta análise confirmam o enunciado de Garcia et al. (2010), em que os processos migratórios entre regiões brasileiras não são uma barreira para o contínuo uso de plantas, assim como de práticas culturais, ao contrário, as migrações encorajam o contato com a rica diversidade biológica e cultural do país e permitem interações interpessoais que contribuem para a transformação das terapias medicinais locais. $\mathrm{O}$ vasto conhecimento dos referidos informantes sobre plantas medicinais sejam elas nativas, cultivadas e/ou do seu antigo local de moradia se explicaria pelas estratégias que estas pessoas tendem a desenvolver quando migram para um novo local, as quais podem ser o fortalecimento da identidade por meio da manutenção de suas práticas culturais e a adaptação cultural ao ambiente ocupado, a partir da absorção de novas práticas (Pieroni \& Vandebroek 2007).

Com relação a variável de gênero, apesar de o teste estatístico não apontar diferenças significativas na riqueza de plantas conhecidas por ambos, notou-se que existem tanto divergências numéricas entre os acervos mencionados por homens e mulheres como na composição das plantas citadas em cada grupo. As mulheres informaram um total de 142 etnoespécies, sendo 31 exclusivamente citadas por elas, enquanto que os homens mencionaram 96 etnoespécies, com 13 exclusivas a este grupo.

No que se refere aos usos das plantas mencionados exclusivamente por homens ou mulheres, notou-se que estas não se destinam a tratar doenças específicas do gênero, mas que tal diferença está mais relacionada ao local onde estas plantas ocorrem. Aquelas citadas apenas pelas mulheres são obtidas em quintais, tais como cariru (Talinum paniculatum (Jacq.) Gaertn.), pariri (Fridericia chica (Bonpl.) L.G.Lohmann), jambu (Acmella oleracea (L.) R.K. Jansen), losna (Artemisia vulgaris L.), melão-de-são-caetano (Momordica charantia L.), vindica (Renealmia alpinia (Rottb.) Maas), oriza (Pogostemon heyneanus Benth) e elixir paregórico (Piper callosum Ruiz \& Pav.). Por outro lado, dentre as espécies mencionadas apenas por homens figuram uxi, cipó-de-fogo (Davilla sp.) e acapú, que por sua vez são coletadas nas áreas de floresta do entorno, assim como unha-de-gato (Uncaria tomentosa (Aubl.) J.F.Gmel), barbatimão e amapá (Parahancornia fasciculata (Poir.) Benoist), frequentemente citadas pelo grupo.
Em pesquisas semelhantes realizadas em comunidades rurais foi observado que embora exista uma semelhança na riqueza de plantas conhecidas pelos diferentes gêneros, isto não significa que o acervo de plantas conhecidas entre homens e mulheres seja similar (Monteiro et al. 2006; Silva et al. 2011). Segundo Voeks (2007), o conhecimento que as mulheres acumulam é derivado da experiência adquirida ao cuidar da saúde de sua família, do maior contato com a agricultura e gestão dos quintais, onde há o cultivo de muitas das espécies citadas exclusivamente por este grupo. Enquanto, os homens, na busca de plantas para construção e combustível, tornam-se os principais conhecedores de plantas obtidas na vegetação secundárias (Costa \& Mitja 2010), além de também conhecerem espécies cultivadas.

Quanto à idade, não houve diferenças significativas na riqueza de plantas conhecidas entre os grupos analisados. Na Figura 2 nota-se que o grupo de pessoas representados pela faixa etária de 58 a 80 anos apresenta um conhecimento sobre plantas medicinais igual ou inferior ao de pessoas das demais classes etárias analisadas. Essa baixa riqueza de plantas conhecidas entre os mais idosos justifica-se pelas limitações que alguns apresentaram em lembrar o nome das

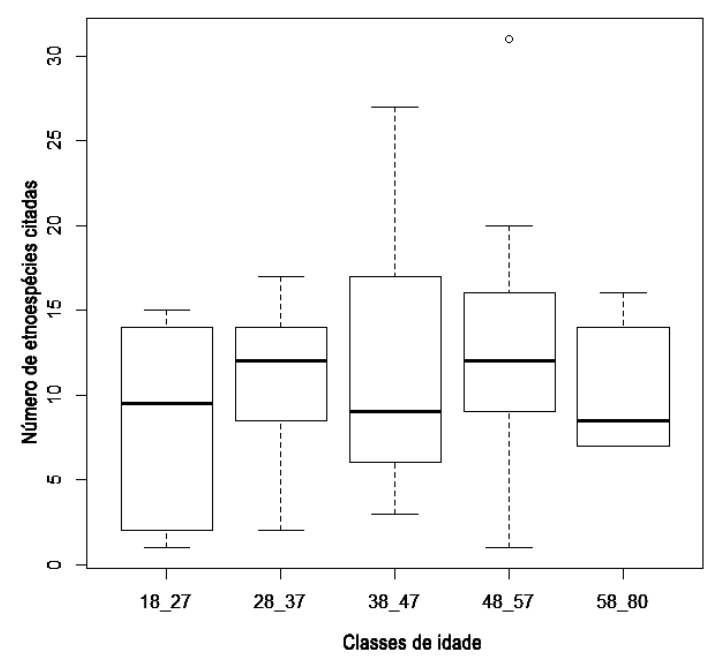

Figura 2 - Distribuição do conhecimento de plantas medicinais entre informantes de diferentes classes de idade no Assentamento rural Paulo Fonteles, Distrito de Mosqueiro, Município de Belém, estado do Pará, Brasil. Figure 2 - Distribution of knowledge of medicinal plants among informants of different age groups in the Paulo Fonteles settlement, Mosqueiro District, Belém, PA. 
plantas e de reconhecê-las na vegetação do entorno. Ademais, observou-se que, tanto os mais jovens como os mais idosos estão envolvidos nas mesmas atividades, sejam elas domésticas, agrícolas ou extrativistas, o que os leva a um acúmulo comum de experiências. Estudos etnobotânicos realizados com outros grupos sociais, entretanto, demonstraram que quanto maior a idade, maior é o acervo de plantas que as pessoas conhecem (Reyes-García et al. 2005; Quilan \& Quilan 2007; Silva et al. 2011; Malthez-Stifel et al. 2012). Em pesquisa recente, Brito et al. (2017a), constataram que apenas a relação entre a idade e o número de plantas citadas não permite fazer inferências sobre a perda de conhecimento em uma comunidade, mas pode indicar que o conhecimento tende a ser transmitido e acumulado gradualmente, o que pode ser observado na presente pesquisa. Estes mesmos autores, assim como outros (Reyes-García et al. 2005; Ayantunde et al. 2008; Silva et al. 2011) observaram que estes conhecimentos tendem a estabilizar ou diminuir após uma certa idade.

\section{Dinâmica do conhecimento}

e uso de plantas medicinais

\section{Usos mantidos}

De acordo com os informantes, 109 das 140 etnoespécies mencionadas eram usadas nos locais onde viviam anteriormente, sejam estes outras cidades do estado do Pará ou outras regiões do Brasil (Tab. S1, disponibilizada no material suplementar <https://doi.org/10.6084/ m9.figshare.13645655.v1>). A frequência de usos mantidos se deve principalmente à disponibilidade da maioria destas no APF e ao fato de que a maioria dos entrevistados é proveniente de outras cidades amazônicas, o que viabiliza o uso contínuo de plantas com usos difundidos na região.

Ao avaliar as plantas que vieram de fora, observou-se que os informantes de outras regiões trouxeram consigo mudas de 22 espécies, entre as quais estão: aroeira (Schinus sp.), ata (Annona squamosa L.), chanãna (Turnera subulata Sm.), pataca (Dillenia indica L) e akòko (Newbouldia laevis (P. Beauv.) Seem), plantas que não são nativas da Amazônia. O uso contínuo de certas espécies por populações migrantes foi um caminho utilizado por Garcia et al. (2010) para evidenciar aquelas que apresentavam alto potencial terapêutico. Isso se torna ainda mais interessante quando estes usos são mantidos mesmo que ocorra exposição a diferentes realidades culturais e ambientais (Volpato et al. 2009).
A fala registrada da informante 01 (50 anos), exemplifica uma espécie que os usos foram mantidos: "Na última vez que fui ao Maranhão trouxe várias mudas de lá para cultivar aqui, porque as plantas de lá uso desde criança e sei que fazem efeito".

Segundo a informante a espécies é a ata (Annona squamosa L.), por considerar a sua eficácia contra diarreias. Esta espécie é originária da Índia, onde também é utilizada, pelas populações locais, para o referido fím, além de muitos outros (Yabesh et al. 2014), tendo seu uso no nordeste brasileiro sido registrado em pesquisas etnobotânicas para fins alimentícios e medicinais (Castro et al. 2011; Albuquerque \& Andrade 2002). A. squamosa possui diversas propriedades terapêuticas atestadas em estudos farmacológicos, dentre as quais figuram sua atividade antimicrobiana e anti-helmíntica, que podem justificar seu uso nesta comunidade, contra diarreias, sintoma comum na presença de microrganismos patógenos (Ma et al. 2017). Por outro lado, caamembeca (Caamembeca spectabilis (DC.) J.F.B.Pastore), cabi (Callaeum antifebrile (Griseb.) D.M.Johnson), cajuí (Anacardium giganteum W.Hancock ex Engl.) e tachi (Tachigali glauca Tul.) tiveram seus usos mantidos, mas não foram encontrados estudos fitoquímicos ou biológicos que atestem suas propriedades terapêuticas.

Além disso, alguns usos tendem a ser mantidos com a finalidade de fortalecer a identidade cultural ou religiosa dos imigrantes, como por exemplo, o uso de akòko, cultivada no quintal do informante 02 (31 anos), onde são realizados cultos de cunho afro-brasileiro, religião que o acompanha desde sua adolescência. Esta espécie é utilizada tanto no cotidiano como nos rituais com a finalidade de melhorar a capacidade mental. Este uso está relacionado à origem africana de akòko e por ser considerada pelos adeptos às religiões afrobrasileiras como um marcador simbólico de lugares sagrados (Voeks 2013). Seus usos medicinais são amplamente difundidos no continente de origem (Sonibare et al. 2009).

\section{Usos incorporados}

Cerca de 61 etnoespécies passaram a ser usadas pelos entrevistados quando eles chegaram no APF (Tab. S1, disponibilizada no material suplementar $<$ https://doi.org/10.6084/m9.figshare.13645655. v1>). A incorporação do uso destas ocorreu por meio de cursos oferecidos pelo MST no período de acampamento, mídias impressas fornecidas 
pelas igrejas locais e informações obtidas com os vizinhos durante a convivência diária e atividades desenvolvidas na mata do entorno. Esta última possibilitou o conhecimento de espécies nativas da região amazônica, principalmente por moradores que vieram de outras regiões do país e trabalhavam no extrativismo e carvoaria. Cita-se como exemplo, o informante 03 (39 anos), oriundo do Piauí e um dos poucos moradores que sabiam encontrar acapu, barbatimão e cajuí, conhecidas através de seus colegas durante o exercício das referidas atividades.

Dentre as 54 espécies, muitas foram utilizadas para tratar doenças previamente conhecidas pelos informantes, que se tornaram mais frequentes quando passaram a residir no APF. Como exemplos destas citam-se açaí, elixir paregórico e escada-dejabuti (Schnella splendens (Kunth) Benth) usadas contra diarreia; algodão roxo e urucum para tratar a gripe; ampicilina (Alternanthera brasiliana (L.) Kuntze) e cajuí como anti-inflamatórios e cicatrizantes. Isto sugere que o uso destas plantas expandiu uma farmacopeia já existente para tratar tais doenças (Medeiros et al. 2012). Outras espécies, por sua vez, foram utilizadas para tratar problemas de saúde acometidos pelos moradores somente no APF, é caso de cajuí, maxixe e caamembeca usadas contra o diabetes, colesterol alto e câncer no útero, respectivamente.

Pesquisadores que têm analisado essas estratégias no uso de plantas por populações migrantes, afirmam que a incorporação de usos só pode ser evidenciada quando um grupo seleciona um recurso medicinal para tratar uma demanda terapêutica desconhecida anteriormente, ocupando assim um nicho na farmacopeia que não era anteriormente considerado (Medeiros et al. 2012). Cajuí e caamembeca são plantas amazônicas (BFG 2018) e tratam diferentes problemas de saúde na região (Milliken 1998; Mors et al. 2000; De Filipps et al. 2004; Coelho-Ferreira 2008, 2009; Berg 2010; Mesquita et al. 2013), entretanto, não foram encontrados nestes registros os usos acima mencionado para estas plantas.

\section{Usos substituídos}

Cerca de 5 etnoespécies utilizadas pelos moradores em suas cidades de origem foram substituídas no APF, seja por sua indisponibilidade atual, ou pela menor eficácia à elas creditada, em relação a outras plantas encontradas no APF (Tab. $\mathrm{S} 1$, disponibilizada no material suplementar $<\mathrm{https}: / /$ doi.org/10.6084/m9.figshare.13645655.v1>). Alguns entrevistados relataram critérios empregados para substituir plantas que utilizavam no seu local de origem, como o informante 03 ( 39 anos) que passou a utilizar cajuí no lugar de aroeira, justificando que ambas atuam como cicatrizantes; a informante 04 (50 anos), que usava inharé (Brosimum sp.) para tratar inflamações uterinas e substituiu por unhade-gato, pela maior eficácia desta; a informante 05 (74 anos), que geralmente usava o eucalipto no combate à gripe no Mato Grosso, mas no APF passou a usar pirarucu, dada a sua maior acessibilidade; e a informante 06 (54 anos), que no Maranhão costumava preparar garrafadas, para tratar inflamação uterina com aroeira, açoita-cavalo e carqueja, mas devido à indisponibilidade dessas plantas, este remédio passou a ser preparado com unha-de-gato e ipê (Handroanthus sp.), sem prejuízo quanto à sua eficácia.

Diversos fatores podem influenciar no processo de substituição, como as semelhanças taxonômicas e morfológicas (Voeks 1990), características químicas e sensoriais das plantas, bem como a influência de contato com pessoas locais (Medeiros et al. 2012). Este último fator desempenha um importante papel nos processos de substituição locais, devido ao habitual compartilhamento de saberes nessas comunidades, característica já mencionada anteriormente. A semelhança taxonômica entre cajuí e aroeira, também pode explicar o uso de uma no lugar da outra, pois ambas pertencem à família Anacardiaceae.

De acordo com Medeiros et al. (2012) espécies com proximidade filogenética podem conter compostos similares, garantindo a sua utilidade para o mesmo propósito. Quanto ao uso de unha-de-gato no lugar de inharé nota-se o resultado de experimentação, comparação e avaliação de eficácia, pois houve a preferência pelo uso da primeira. Tanto o eucalipto como o pirarucu fazem parte de um sistema de plantas cultivadas em várias partes do Brasil para atender a uma demanda comum de doenças, o que justifica o seu processo de substituição. No terceiro caso, em que há substituição da carqueja, aroeira e açoita-cavalo por espécies nativas, embora as três plantas sejam comercializadas nos mercados da região metropolitana de Belém, o acesso aos mesmos é limitado pela distância e dificuldades econômicas. Vale ressaltar que a maior parte das plantas substitutas é proveniente do extrativismo na área de preservação, o que evidencia a importância desta na manutenção do uso de plantas medicinais diante de um processo de adaptação a uma nova realidade ambiental e social. 


\section{Usos descontinuados}

$\mathrm{Na}$ categoria de uso descontinuado cerca de 25 etnoespécies entraram em desuso para alguns dos informantes (Tab. S1, disponibilizada no material suplementar $<$ https://doi.org/10.6084/ m9.figshare.13645655.v1>). O abandono do uso destas plantas foi associado principalmente à sua indisponibilidade ou à dificuldade de encontrá-las no atual local de moradia, seguido da não ocorrência de doenças para as quais estas eram empregadas. Citamse como exemplos, mussambê (Tarenaya sp.), cujas folhas eram utilizadas na preparação de chás para inalação contra gripes e resfriados; crista-de-galo (Heliotropium sp.), da qual se retiravam as folhas para compor um emplastro para atuar no processo de cicatrização de feridas; japecanga (Smilax sp.), cuja raiz era utilizada para tratar sífilis; batata-de-purga/ batatão (Operculina sp.) usada como um purgativo e contra vermes e angico (Anadenanthera sp.), da qual se retirava o sumo da entrecasca para a preparação de emplastros e xaropes contra ferimentos e gripes. Estas duas últimas também foram mencionadas entre as plantas com usos descontinuados na pesquisa realizada em São Paulo entre imigrantes de outros estados do Brasil (Garcia et al. 2010).

\section{Conclusão}

Esta pesquisa demonstrou que o conhecimento sobre plantas medicinais no Assentamento Paulo Fonteles encontra-se em um processo dinâmico de aquisição, perdas e adequações, resultante tanto da heterogeneidade de origem dos informantes, como dos diversos distúrbios ocorridos ao longo dos períodos que antecedem e sucedem a formação do assentamento. Contudo, as plantas medicinais constituem um recurso prioritário nos cuidados de saúde locais.

Embora, fatores como gênero e idade não tenham influenciado significativamente na riqueza de plantas conhecidas entre os informantes, o presente estudo aponta que homens e mulheres conhecem e utilizam diferentes repertórios de plantas e que este conhecimento está sendo difundido entre indivíduos de diferentes faixas etárias existentes na comunidade. No que se refere à origem, a migração dos informantes que vieram de outros estados do Brasil contribuiu significativamente para a ampliação do conhecimento sobre plantas medicinais. Além disso, o aspecto migratório não foi uma barreira para o uso contínuo de plantas do local de origem dos informantes, ao contrário propiciou o incremento da diversidade flora local e a valorização de plantas nativas da Amazônia.

\section{Agradecimentos}

Agradecemos aos moradores do Assentamento Paulo Fonteles, que voluntariamente concordaram em participar deste estudo, compartilhando seus conhecimentos sobre plantas medicinais e contribuindo para a elaboração e implementação do mesmo. Agradecemos também ao Conselho Nacional de Desenvolvimento Científico e Tecnológico (CNPq), a bolsa de Pesquisa de Mestrado desenvolvida no âmbito do Programa de Pós-graduação em Ciências Biológicas Botânica Tropical da Universidade Federal Rural da Amazônia e Museu Paraense Emílio Goeldi (UFRA/MPEG); à Vice-Presidência de Ambiente, Atenção e Promoção à Saúde, da Fundação Oswaldo Cruz (VPAAPS-Fiocruz), que apoiou o presente trabalho através do Projeto Rede Experiências, Tecnologias e Inovação em Saúde - RetisFito; e ao Programa de Capacitação Institucional (MPEG/MCTIC), a vigente bolsa ao primeiro autor.

\section{Referências}

Albuquerque UP \& Andrade LHC (2002) Uso de recursos vegetais da Caatinga: o caso do agreste do estado de Pernambuco (nordeste do Brasil). Interciencia 27: 336-345.

Albuquerque UP, Ramos MA, Lucena RFP \& Alencar NL (2014) Methods and techniques used to collect ethnobiological data. In: Albuquerque UP, Cunha LVFC, Lucena RFP \& Alves RRN (ed.) Methods and techniques in ethnobiology and ethnoecology. Springer, New York. Pp. 15-37.

Almeida MZ (2011) Plantas Medicinais. $3^{\text {a }}$ ed. EDUFBA, Salvador. $35 \mathrm{p}$.

Almeida LS \& Gama JRV (2014) Quintais agroflorestais: estrutura, composição florística e aspectos socioambientais em área de assentamento rural na Amazônia brasileira. Ciência Florestal, Santa Maria 24: 1041-1053.

Alexiades M (1996) Selected guidlines for ethnobotanical research: a field manual. The New York Botanical Garden, New York. Pp. 55-67.

Araújo MM (2009) Estudo etnobotânico das plantas utilizadas como medicinais no Assentamento Santo Antonio, Cajazeiras, Pb, Brasil. Patos. Dissertação de Mestrado. Universidade Federal de Campina Grande, Campina Grande. 125p.

Ayantunde AA, Briejer M, Hiernaux P, Udo HMJ \& Tabo $\mathrm{R}$ (2008) Botanical knowledge and its differentiation by age, gender and ethnicity in southwestern Níger. Journal of Human Ecology 36: 881-889.

Belo DC \& Pedlowski MA(2014) Acampamentos do MST e sua importância na formação da identidade do Sem Terra. Revista Nera 24: 71-85. 
Berg MEVD (2010) Plantas medicinais na Amazônia: contribuição ao seu conhecimento sistemático $3^{\mathrm{a}}$ ed. Museu Paraense Emílio Goeldi, Belém. 268p.

BFG - The Brazil Flora Group (2018) Brazilian Flora 2020: innovation and collaboration to meet Target 1 of the Global Strategy for Plant Conservation (GSPC). Rodriguésia 69: 1513-1527.

Brasil (2015) Lei $\mathrm{n}^{\mathrm{o}} 13.123$, de 20 de maio de 2015. Regulamenta o inciso II do $\S 1^{\circ}$ e o $\S 4^{\circ}$ do art. 225 da Constituição Federal, o Artigo 1, a alínea j do Artigo 8, a alínea c do Artigo 10, o Artigo 15 e os $\S \S$ $3^{\circ}$ e $4^{\circ}$ do Artigo 16 da Convenção sobre Diversidade Biológica, promulgada pelo Decreto $\mathrm{n}^{\circ} 2.519$, de 16 de março de 1998; dispõe sobre o acesso ao patrimônio genético, sobre a proteção e o acesso ao conhecimento tradicional associado e sobre a repartição de benefícios para conservação e uso sustentável da biodiversidade; revoga a Medida Provisória $n^{\circ}$ 2.186-16, de 23 de agosto de 2001; e dá outras providências. Disponível em $<$ http:/www.planalto.gov.br/ccivil_03/_ato20152018/2015/lei/113123.htm>. Acesso em 17 junho 2019.

Brito CC, Silva TC, Albuquerque UP, Ramos MA, Ferreira Júnior WS, Barros FN, Neto EMC \& Medeiros PM (2017a) The use of different indicators for interpreting the local knowledge loss on medical plants. Revista Brasileira de Farmacognosia 27: 245-250.

Brito MFM, Marín EA \& Cruz DD (2017b) Plantas medicinais nos assentamentos rurais em uma área de proteção no litoral do Nordeste brasileiro. Ambiente \& Sociedade 1: 83-104.

Byng JW, Chase MW, Christenhusz MJ, Fay MF, Judd WS, Mabberley DJ, Briggs B, Soltis DE, Sennikov AN, Soltis PS \& Stevens PF (2016) An update of the Angiosperm Phylogeny Group classification for the orders and families of flowering plants: APG IV. Botanical Journal of the Linnean Society 181: 1-20.

Caldart RS (2004) Pedagogia do Movimento Sem Terra. Expressão Popular, São Paulo. Pp 177 - 179.

Castro JA, Brasileiro BP, Lyra DH, Pereira DA, Chaves JL \& Amaral CLF (2011) Ethnobotanical study of traditional uses of medicinal plants: the flora of caatinga in the community of Cravolândia-BA. Brazil Journal of Medicinal Plants Research 5: 1905-1917.

Chambers JM (1998) Programming with data. Springer, New York.

Coelho-Ferreira MR (2008) Notas etnobotânicas sobre as plantas medicinais. In: Jardim MAG \& Zoghbi MGB (eds.) A flora da Resex Chocoaré-Mato Grosso (PA): diversidade e usos. MPEG, Belém. Pp. 63-90.

Coelho-Ferreira MR (2009) Medicinal knowledge and plant utilization in an Amazonian coastal community of Marudá, Pará state, Brazil. Journal of Ethnopharmacology 126: 159-175.

Costa JR \& Mitja D (2010) Uso dos recursos vegetais por agricultores familiares de Manacapuru, AM. Acta Amazonica 40: 49-58.

Cunha SA \& Bortolotto IM (2011) Etnobotânica de plantas medicinais no Assentamento Monjolinho, município de Anastácio, Mato Grosso do Sul, Brasil. Acta Botanica Brasilica 3: 685-698.

De Filipps RA, Maina SL \& Crepin J (2004) Medicinal plants of the Guianas (Guyana, Surinam, French Guiana). Smithsonian Institute, Washington, DC. $477 \mathrm{p}$.

Ezzine-de-Blas D, Borner J, Violato-Espada AL, Nascimento N \& Piketty MG (2011) Forest loss and management in land reform settlements: Implications for REDD governance in the Brazilian Amazon. Environmental Science \& Policy 14: 188-200.

Ferreira Junior WS, Nascimento ALB, Ramos LA, Medeiros PM, Soldati GT \& Albuquerque UP (2013) Resiliência e adaptação em sistemas socioecológicos. In: Albuquerque UP (ed.) Etnobiologia: bases ecológicas e evolutivas. NUPEA, Recife. Pp. 67-84.

Figueiredo GC \& Pinto JMR (2014) Acampamento e assentamento: participação, experiência e vivência em dois momentos da luta pela terra. Psicologia \& Sociedade 26: 562-571.

Freitas JC \& Fernandes MEB (2006) Uso de plantas medicinais na comunidade Enfarrusca, Bragança, Pará. Boletim do Museu Paraense Emílio Goeldi 1: 11-26.

Garcia D, Domingues MV \& Rodrigues E (2010) Ethnopharmacological survey among migrants living in the Southeast Atlantic Forest of Diadema, São Paulo, Brazil. Journal of Ethnobiology and Ethnomedicine 6: 1-19.

Gavioli FR (2012) Agrobiodiversidade e manejo de recursos locais no assentamento rural Monte Alegre, SP, Brasil. Revista de Diálogo e Desconexão 5: 1.

Geilfus F (2002) 80 herramientas para o desarrollo participativo: diagnóstico, planificación, monitoreo, evaluación. Prochalate-Iica-Holanda/Laderas CA, San Salvador. 197p.

Guerra AMNM, Pessoa MF, Souza CSM \& Maracajá PB (2010) Utilização de plantas medicinais pela comunidade rural Moacir Lucena, Apodi-RN. Bioscience Journal, Uberlandia 26: 442-450.

Inta A, Shengji P, Balslev H, Wangpakapattanawong P \& Trisonthi C (2008) A comparative study on medicinal plants used in Akha's traditional medicine in China and Thailand, cultural coherence or ecological divergence? Journal of Ethnopharmacology 116: 508-517.

Livro Vermelho da Flora do Brasil (2013) Texto e organização de Martinelli G \& Moraes MA. Andrea Jakobsson, Instituto de Pesquisas Jardim Botânico do Rio de Janeiro, Rio de Janeiro. 1100p.

Ma C, Chen Y, Chen J, Li X \& Chen Y (2017) A review on Annona squamosa L.: phytochemicals and biological activities. The American Journal of Chinese Medicine. 45: 1-32.

Martins AG, Rosário DL, Barros MN \& Jardim MAG (2005) Levantamento etnobotânico de plantas medicinais, alimentares e tóxicas da Ilha do Combú, município de Belém, estado do Pará, Brasil. Revista 
Brasileira de Farmacognosia 86: 21-30.

Matos SA (2011) Plantas medicinais dos quintais de agricultores familiares do Travessão 338 Sul, Pacajá, Pará, Brasil: uso, conhecimento e valorização. Dissertação de Mestrado. Universidade Federal Rural da Amazônia, Belém. 107p.

Malthez-Stifel S, Brandt R, Lachmuth S \& Rist S (2012) Are the young less knowl-edgeable? Local knowledge of natural remedies and its transformations in the Andean Highlands. Human Ecology 40: 909-930.

Medeiros PM, Almeida ALS, Lucena RFP, Souto FJB \& Albuquerque (2010) Uso de estímulos visuais na pesquisa etnobiológica. In: Albuquerque UP, Lucena RFP \& Cunha LVFC (orgs.) Métodos e técnicas na pesquisa etnobiológica e etnoecológica. NUPPEA, Recife. Pp. 153-169.

Medeiros PM, Soldati GT, Alencar NL, Vandebroek I, Pieroni A, Hanazaki N \& Albuquerque UP (2012) The use of medicinal plants by migrant people: adaptation, maintenance, and replacement. Evidence-Based Complementary and Alternative Medicine 2012: 1-11.

Milliken W (1998) Plantas medicinais, malária e povos indígenas: estudos etnobotânicos no norte da Amazônia. Boletim do Museu Integrado de Roraima 4: 23-30.

Missouri Botanical Garden (2018) Disponível em $<$ http:// www.tropicos.org/>. Acesso em 30 maio 2018.

Monteiro JM, Almeida CFCBR, Albuquerque UP, Lucena RFP, Florentino ATNF \& Oliveira RLC (2006) Use and traditional management of Anadenanthera colubrine (Vell.) Brenan in the semi-arid region of northeastern Brazil. Journal of Ethnobiology and Ethnomedicine 2: 1-7.

Mors WB, Rizzini CT \& Pereira NA (2000) Medicinal plants of Brazil. Reference Publications, Algonac. $501 \mathrm{p}$.

Nesheim I, Dhillion SS \& Stølen KA (2006) What happens to traditional knowledge and use of natural resources when people migrate? Human Ecology 34: 99-131.

Oliveira PS (2010) Plantas medicinais numa comunidade rural assentada no município de CordeirópolisSP: etnofarmacologia e educação. Dissertação de Mestrado. Universidade Estadual de Campinas, Campinas. 93p.

Pantoja RCP (2010) Ação coletiva na criação e gestão do projeto de assentamento Paulo Fonteles em Mosqueiro, Belém, Pará. Dissertação de Mestrado. Universidade Federal do Pará, Belém. 95p.

Pieroni A \& Vandebroek I (2007) Traveling plants and cultures. The ethnobiology and ethnopharmacy of migrations. Berghahn, Oxford. Pp: 1-13.

Quilan MB \& Quilan RJ (2007) Modernization and medicinal plant knowledge in a Caribbean horticultural village. Medical Anthropology Quarterly 21: 169-192.

Reyes-García V, Vadez V, Byron E, Apaza L, Leonard WR, Perez E \& Wilkie D (2005) Market economy and the loss of folk knowledge of plant uses: estimates from the Tsimane' of the Bolivian Amazon. Current Anthropology 46: 651-656.

Scopinho RA (2010) Condições de vida e saúde do trabalhador em assentamento rural. Ciência \& Saúde Coletiva 15: 1575-1584.

Silva FS, Ramos MA, Hanazaki N \& Albuquerque UP (2011) Dynamics of traditional knowledge of medicinal plants in a rural community in the Brazilian semi-arid region. Revista Brasileira de Farmacognosia 21: 382-391.

Soldati GT, Hanazaki N, Crivos M \& Albuquerque UP (2015) Does environmental instability favor the production and horizontal transmission of knowledge regarding medicinal plants? A study in Southeast Brazil. Plos One 10: 126-389.

Sonibare MA, Moody JO \& Adesanya EO (2009) Use of medicinal plants for the treatment of measles in Nigeria. Journal of Ethnopharmacology: 268-272.

The Plant List (2019) Disponível em <http://www. theplantlist.org/>. Acesso em 06 junho 2019.

Tunholi VP; Ramos MA; ScariotA. (2013). Availability and use of woody plants in a agrarian reform settlement in the cerrado of the state of Goiás, Brazil. Acta Botanica Brasilica 27: 604-612.

Visbiski VN, Neto PHW \& Santos AL (2003) Uso popular da plantas medicinais no assentamento Guanabara, Imbaú, PR. Ciências Exatas da Terra 9: 13-20.

Voeks RA(1990) "Sacred leaves of Brazilian Candomble". Geographical Review 80: 118-131.

Voeks RA(2007) Are women reservoirs of traditional plant knowledge? Gender, ethnobotany and globalization in northeast Brazil. Singapore Journal of Tropical Geography 28: 7-20.

Voeks RA(2013) Ethnobotany of Brazil's African diaspora: the role of floristic homogenization. In: Voeks R \& Rashford J (eds.) African ethnobotany in the Americas. Springer, New York. Pp. 395-415.

Volpato G, Godínez D, Beyra A \& Barreto A (2009) Uses of medicinal plants by Haitian immigrants and their descendants in the Province of Camagüey, Cuba. Journal of Ethnobiology and Ethnomedicine 5: 1-9.

Yabesh JEM, Prabhu S \& Vijayakumar S (2014) An ethnobotanical study of medicinal plants used by traditional healers in silent valley of Kerala, India. Journal of Ethnopharmacology 154: 774-89.

Waldstein A(2006) Mexican migrant ethnopharmacology: Pharmacopoeia, classification of medicines and explanations of efficacy. Journal of Ethnopharmacology 108: 299-310. 